\title{
Severe thrombocytopaenia in patients with vivax malaria compared to falciparum malaria: a systematic review and meta- analysis
}

Cho Naing ${ }^{1,2^{*}}$ (D) and Maxine A. Whittaker ${ }^{2}$

\begin{abstract}
Background: Plasmodium vivax is the most geographically widespread species among human malaria parasites. Immunopathological studies have shown that platelets are an important component of the host innate immune response against malaria infections. The objectives of this study were to quantify thrombocytopaenia in $P$. vivax malaria patients and to determine the associated risks of severe thrombocytopaenia in patients with vivax malaria compared to patients with $P$. falciparum malaria.
\end{abstract}

Main body: A systematic review and meta-analysis of the available literature on thrombocytopaenia in $P$. vivax malaria patients was undertaken. Relevant studies in health-related electronic databases were identified and reviewed. The Preferred Reporting Items for Systematic Reviews and Meta-Analyses guidelines were followed. Fifty-eight observational studies $(n=29664)$ were included in the current review. Severe thrombocytopaenia $\left(<50000 / \mathrm{mm}^{3}\right)$ to very severe thrombocytopaenia $\left(<20000 / \mathrm{mm}^{3}\right)$ was observed in $10.1 \%$ of patients with P. vivax infection. A meta-analysis of 11 observational studies showed an equal risk of developing severe/very severe thrombocytopaenia between the patients with $P$. vivax malaria and those with $P$. falciparum malaria (OR: $1.98,95 \%$ Cl: $0.92-4.25$ ). This indicates that thrombocytopaenia is as equally a common manifestation in $P$. vivax and $P$. falciparum malaria patients. One study showed a higher risk of developing very severe thrombocytopaenia in children with severe P. vivax malaria than with severe P. falciparum malaria (OR: 2.80, 95\% Cl: 1.48-5.29). However, a pooled analysis of two studies showed an equal risk among adult severe cases (OR: $1.19,95 \%$ Cl: $0.51-2.77$ ). This indicates that the risk of developing thrombocytopaenia in P. vivax malaria can vary with immune status in both children and adults. One study reported higher levels of urea and serum bilirubin in patients with $P$. vivax malaria and severe thrombocytopaenia compared with patients mild thrombocytopaenia or no thrombocytopaenia, $(P<0.001$ in all comparisons). A pooled analysis of two other studies showed a similar proportion of bleeding episodes with thrombocytopaenia in severe $P$. vivax patients and severe $P$. falciparum patients $(P=0.09)$. This implied that both $P$. vivax and $P$. falciparum infections could present with bleeding episodes, if there had been a change in platelet counts in the infected patients. A pooled analysis of another two studies showed an equal risk of mortality with severe thrombocytopaenia in both $P$. vivax and $P$. falciparum malaria patients (OR: 1.16, 95\% Cl: 0.30-4.60). However, due to the low number of studies with small sample sizes within the subset of studies that provided clinically relevant information, our confidence in the estimates is limited.

(Continued on next page)

\footnotetext{
* Correspondence: cho3699@gmail.com

${ }^{1}$ Institute for Research, Development and Innovation (IRDI), International Medical University, Kuala Lumpur, Malaysia

${ }^{2}$ College of Public Health, Medical and Veterinary Sciences, James Cook

University, Queensland, Australia
} 
(Continued from previous page)

Conclusion: The current review has provided some evidence of the clinical relevance of severe thrombocytopaenia in $P$. vivax malaria. To substantiate these findings, there is a need for well designed, large-scale, prospective studies among patients infected with $P$. vivax. These should include patients from different countries and epidemiological settings with various age and gender groups represented.

Keywords: Thrombocytopaenia, Malaria, Plasmodium vivax, Systematic review

\section{Multilingual abstracts}

Please see Additional file 1 for translation of the abstract into the six official working languages of the United Nations.

\section{Background}

Plasmodium vivax is the most geographically widespread species of human malaria parasites, affecting almost $40 \%$ of the world's population [1-3]. The invisibility of the dormant liver stages of $P$. vivax to any diagnostic method may lead to an underestimation of the true prevalence of $P$. vivax far more than $P$. falciparum. This means $P$. vivax parasites could be highly prevalent in many endemic settings [3]. Most recorded $P$. vivax malaria cases originate from South East Asia and the Western Pacific, and a significant number also occur in Africa and South America [1-4]. Immunopathological studies have shown that platelets are an important component of the host innate immune responses against malaria infection $[5,6]$. Thrombocytopaenia (platelet count $<150000 / \mathrm{mm}^{3}$ ) appears to be a very frequent haematological alteration in acute malaria infections $[2,5-7]$. Severe malaria is defined by a demonstration of asexual forms of the malaria parasites in the blood of a patient with a potentially fatal manifestation or complication of malaria in whom other diagnoses have been excluded $[4,8]$. In brief, the clinical features of severe falciparum malaria are impaired consciousness, prostration, multiple convulsions, acute respiratory distress, circulatory collapse or shock, acute kidney injury, clinical jaundice and abnormal bleeding [8]. Although thrombocytopaenia is not included in the current World Health Organization (WHO) criteria for defining severe $P$. falciparum malaria [8], its clinical importance has been widely recognized. For instance, a retrospective analysis on 614 patients has shown that multi-organ dysfunction (MOD) and subsequent risk of death were found in patients with thrombocytopaenia [9]. However similar information on $P$. vivax malaria is limited. Since the life cycle and pathophysiology of $P$. falciparum and $P$. vivax infections are different, it is important to understand the role of platelets in P. vivax malaria.

There has been a recent increase in studies in the literature which have assessed the frequency of thrombocytopaenia in $P$. vivax infection. These studies varied with respect to geographical location, thrombocytopaenia grading, study design and other variables. Understanding the occurrence and role of thrombocytopaenia in $P$. vivax malaria may improve clinical practice guidelines. The aim of this study was to undertake a critical analysis of the available literature on thrombocytopaenia in $P$. vivax malaria. The overall objectives of this study were to quantify thrombocytopaenia in P. vivax malaria patients and to establish the associated relative risks of severe thrombocytopaenia in patients with $P$. vivax malaria compared to patients with $P$. falciparum malaria.

\section{Methods}

The current review was done, following the Preferred Reporting Items for Systematic Reviews and Meta-Analyses (PRISMA) guideline [10] (Additional file 2).

\section{Literature search}

One investigator searched for the original studies, addressing thrombocytopaenia in human $P$. vivax malaria. A literature search in electronic database of MEDLINE (1946 to October 2017), EMBASE (1947 to October 2017), Google Scholar, the Latin American and Caribbean Health Sciences Literature (LILACS) and African Journals Online (AJOL). The following Medical Subject Heading (MeSH) terms were used: "(vivax malaria OR vivax OR Plasmodium vivax OR falciparum malaria OR falciparum OR Plasmodium falciparum)" combined with "(platelets OR platelet counts OR thrombocytopaenia OR thrombocytopenia)." The search strategy was slightly adjusted according to the requirements of different databases. The search was limited to studies published in English until October 2017. We also checked the references of retrieved articles and relevant reviews for any additional studies.

Studies were selected if they (i) had $\geq 10$ participants infected with $P$. vivax, residing in malaria endemic countries; (ii) were observational studies, (iii) provided data on frequency of thrombocytopaenia in $P$. vivax infection, compared frequency/risk of thrombocytopaenia between $P$. vivax and $P$. falciparum infection or between severe vivax malaria and uncomplicated vivax malaria/healthy controls, and (iv) provided relative risk (RR) or odds ratio (OR) estimates with its $95 \%$ confidence interval $(C D)$ (or raw data to compute the estimates) of the association between $P$. vivax and $P$. falciparum or between severe vivax malaria and 
uncomplicated vivax malaria/healthy controls. If more than one study presented data from the same participants, the study with more complete information was used. Studies not meeting the above criteria were excluded. Studies assessing a special group of the population such as imported malaria or pregnant women were also excluded. This is because acquisition of immunity to malaria in people with imported malaria is different from those residing in endemic countries. Also, physiological changes in pregnant women are different from non-pregnant population.

Thrombocytopaenia was further classified into mild $\left(100-150000 / \mathrm{mm}^{3}\right)$, moderate $\left(50-100000 / \mathrm{mm}^{3}\right)$ [11], severe $\left(<50000 / \mathrm{mm}^{3}\right)$ and very severe thrombocytopaenia $\left(<20000 / \mathrm{mm}^{3}\right)$ [12]. The platelet count or grading used in the analysis was as reported by the authors in their published work. Parasitological diagnosis of malaria was made based on microscopy of Giemsa-stained thick/thin blood films (TBF), rapid onsite diagnostic test (RDT) or any diagnostic technique (e.g. QBC), regardless of subsequent PCR-based confirmation of the species. Severe/complicated $P$. vivax malaria and uncomplicated/nonsevere $P$. vivax malaria in the current analysis were as described by the authors in the primary studies $[12,46]$. The current review considered only patients with monoinfections, so patients with mixed infections were excluded.

\section{Data extraction and data synthesis}

Two investigators individually screened the titles and abstracts, and then selected full-text articles, according to the inclusion criteria. The two investigators independently extracted information from each included study using a data extraction form prepared for this meta-analysis. This data extraction form had been pre-tested by the investigators on a sample of papers to check its utility, comprehensiveness and ease of use. Information collected was author, year of publication, country, patient characteristics (e.g. mean age, gender), study characteristics (e.g. sample size, study design, settings) and the reported clinical outcomes. Disagreements between the two investigators were resolved by consensus.

To assess the pooled proportions of thrombocytopaenia in $P$. vivax malaria, proportion data was extracted from the included studies. Raw cell counts were extracted from the studies and Freeman-Tukey double arcsine transformation and exact $C I$ were employed with the individual studies for that purpose. Meta-analysis of observational studies, which assessed the risk associated with severe thrombocytopaenia in $P$. vivax malaria, was performed. The odds ratio $(O R)$ of the pooled analysis was used to compare dichotomous data [13]. For the studies with mean and standard deviation (SD) of platelet counts, the mean difference (MD) and its 95\% CI for the pooled analysis were used. When an individual study provided mean value and range, the range was transformed to SD through the formula $\mathrm{SD}=(($ maximum-minimum $) / 4)$. If the studies provided median and interquartile ranges, the data was transformed into mean and SD, using a formula developed by Hozo and associates [14]. The $I^{2}$ test was used for the assessment of statistical heterogeneity between studies. A value of $I^{2}>50 \%$ indicated substantial heterogeneity [15]. The random effects model was employed as there was substantial heterogeneity between studies. Data analysis was done by STATA 14.0 (STATACorp, Txt, USA).

\section{Results}

Figure 1 provides a four-phase flow chart of the study selection process. The initial search yielded 1025 citations. After the title and abstract screening, 77 full-text papers were reviewed and a final selection of 58 studies (a total of 5536 patients had thrombocytopaenia amongst a total of 29664 patients investigated) met the pre-specified inclusion criteria $[11,12,16-71]$. A summary of the 19 excluded studies $[7,72-89]$ is provided in Additional file 3.

\section{Characteristics of the included studies}

All studies included in the present analysis used observational designs. Twenty-nine studies ( $50 \%$ of the included 58 studies) were prospective studies. Of them, the vast majority of participants infected with $P$. vivax $(66 \%, 19552 /$ 29664) were from a single large study conducted in Indonesia [11]. Half of the studies were conducted in India

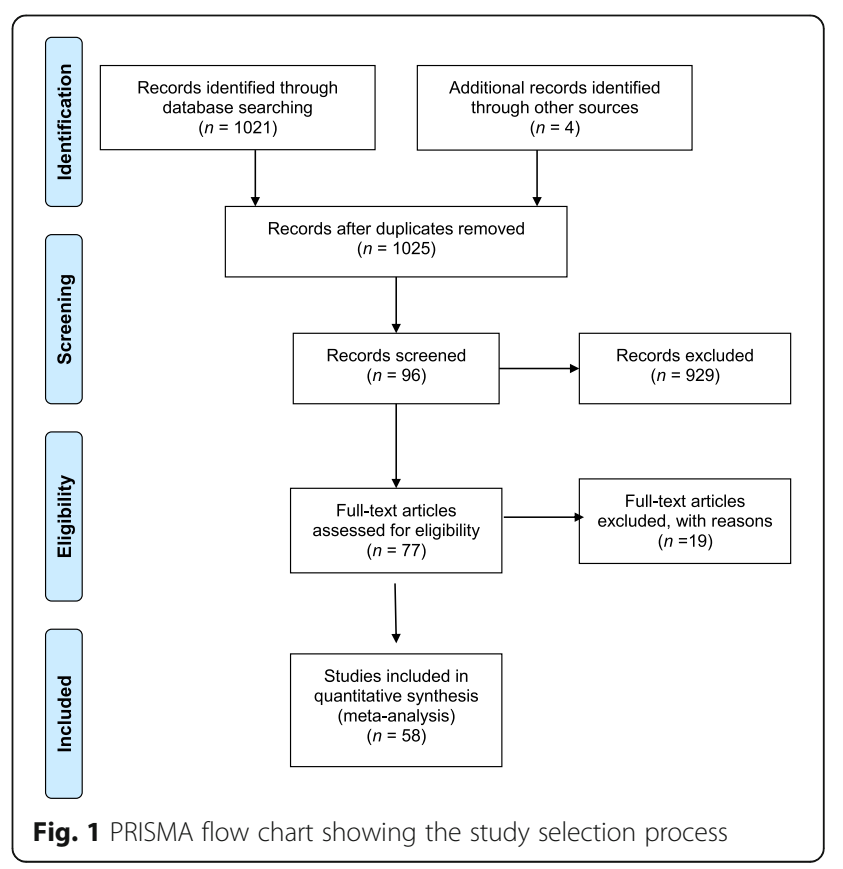


(50\%) and the study period covered the years 1988-2015. Only $25 \%$ of the included studies had PCR confirmation of parasite species (Additional file 4).

Overall, a pooled analysis showed that $18.7 \%$ (5536/ 29 664, 95\% CI: 17.0-20.2\%) of patients with P. vivax malaria had platelet counts $<150000 / \mathrm{mm}^{3}$. Of these, $10.1 \%$ (2674/23 412, 95\% CI: 9.0-10.8\%) of the patients infected with $P$. vivax had either severe thrombocytopaenia (9\%) or very severe thrombocytopaenia (13\%) (Fig. 2). The proportions of mild and moderate thrombocytopaenia are presented in Additional file 5. There was an absence of statistical heterogeneity $\left(I^{2}: 0 \%\right)$, suggesting a high level of homogeneity between studies in each gradient of thrombocytopaenia.

A subgroup analysis of 11 studies $(11,12,20,22,30,33$, $38,55,66,69,70)$ showed patients with $P$. vivax malaria (1930/ 20 863) and those with P. falciparum malaria (5895/ 38450$)$ had an equal risk of developing severe/very severe thrombocytopaenia, (OR: 1.98, 95\% CI: 0.92-4.25) (Table 1). In a subgroup of 3 studies, a pooled analysis showed patients with severe $P$. vivax malaria and those with severe $P$. falciparum malaria had an equal risk of developing severe/very severe thrombocytopaenia [12, 20, 38]. Further stratification by age groups showed children with severe $P$. vivax malaria (60/278) had a higher risk of developing very severe thrombocytopaenia than in those with severe P. falciparum malaria (13/145) (OR: 2.80, 95\% CI: 1.48-5.29) [12]. However an equal risk was observed for adult severe cases with $P$. vivax malaria and those with P. falciparum malaria (45/186 vs $70 / 207$, OR: 1.19 , $95 \%$ CI: 0.51-2.77, $P=0.22$ ) [20, 38] (Fig. 3).

A subset of 4 studies [24, 35, 41, 58] reported the mean platelet counts. Decreased platelet counts were recorded in patients with $P$. vivax malaria compared to the healthy controls $(P<0.001$ in all four studies $)$ (Additional file 6). In another subgroup of 2 studies with PCR confirmed malaria [12, 29], the mean platelet counts were almost the same in both uncomplicated $P$. vivax malaria and uncomplicated $P$. falciparum malaria (MD: -24.5, 95\% CI: $-57.8-8.6, P=0.15)$. However, one study on severe malaria [46] showed a relatively lower mean platelet count in patients with severe $P$. falciparum malaria than in those with severe $P$. vivax malaria (MD: 46.0, 95\% CI: 37.5-54.5, $P<0.0001)$. This implied that the platelet counts could vary with severity of malaria.

A pooled analysis of another 4 studies [12, 32, 59, 63] showed that $15 \%$ (95\% CI: 9-21\%) of P. vivax malaria patients with thrombocytopaenia developed

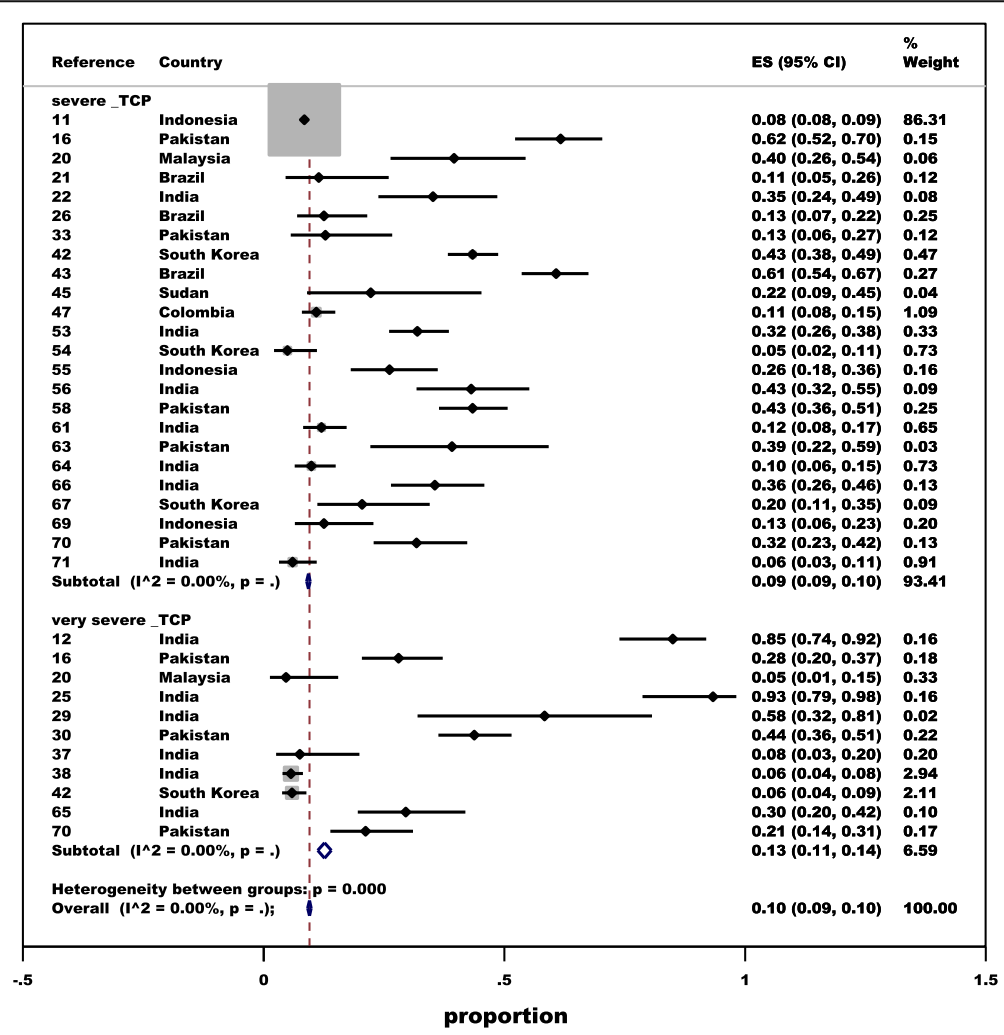

Fig. 2 Proportion of severe to very severe thrombocytopaenia in patients with vivax malaria. TCP: Thrombocytopenia; ES: Effect estimate. The grey zone indicates a very narrow 95\% upper and lower bound. The size of these grey areas reflects the weightage of their contribution to the pooled analysis. $P$ value is not indicated by default, when $P^{2}$ value becomes $0 \%$ 
Table 1 Distribution of thrombocytopaenia between vivax malaria and falciparum malaria

\begin{tabular}{|c|c|c|c|c|c|c|c|}
\hline \multirow[t]{2}{*}{ Study [Reference] } & \multirow{2}{*}{$\begin{array}{l}\text { thrombocytopaenia } \\
\text { grade }\end{array}$} & \multicolumn{2}{|c|}{ Vivax malaria } & \multicolumn{2}{|c|}{ Falciparum malaria } & \multirow{2}{*}{$\begin{array}{l}\text { Odds } \\
\text { ratio }\end{array}$} & \multirow[t]{2}{*}{$95 \% \mathrm{Cl}$} \\
\hline & & event & total & event & total & & \\
\hline Taylor, 2008 [69] & severe & 8 & 64 & 5 & 98 & 2.65 & $0.83-8.52$ \\
\hline Poespoprodjo, 2009 [55] & severe & 23 & 88 & 5 & 51 & 3.25 & $1.15-9.19$ \\
\hline Khan, 2012 [33] & severe & 5 & 39 & 22 & 82 & 0.4 & $0.14-1.15$ \\
\hline Dhungat, 2013 [22] & severe & 19 & 54 & 13 & 28 & 0.62 & $0.24-1.58$ \\
\hline Barber, 2013 [20] & severe & 19 & 43 & 61 & 122 & 0.79 & $0.39-1.59$ \\
\hline Singh, 2014 [66] & severe & 32 & 90 & 32 & 90 & 1 & $0.54-1.84$ \\
\hline Haroon, 2013 [30] & severe & 70 & 110 & 2 & 472 & 411.2 & $97.2-1739.5$ \\
\hline Lampah, 2015 [11] & severe & 1650 & 19552 & 5722 & 36759 & 0.49 & $0.47-0.52$ \\
\hline Tanwar, 2012 [12] & very severe & 60 & 278 & 13 & 145 & 2.79 & $1.47-5.28$ \\
\hline Kochar, 2010b [38] & very severe & 26 & 460 & 9 & 525 & 3.43 & $1.59-7.4$ \\
\hline Uttra, 2010 [70] & very severe & 18 & 85 & 11 & 78 & 1.64 & $0.72-3.72$ \\
\hline Pooled, Odds Ratio & $P=94.1 \%$ & & & & & 1.98 & $0.92-4.25$ \\
\hline
\end{tabular}

minor bleeding episodes (Additional file 7). These bleeding manifestations were epistaxis, haematemesis, petechiae and purpura. A pooled analysis of 2 studies $[12,37]$ showed a similar risk of developing bleeding episodes for both thrombocytopaenia with severe $P$. vivax malaria $(15.7 \%, 70 / 445)$ and with $P$. falciparum malaria (11.2\%, 30/269) (OR: 0.67, 95\% CI: 0.43-1.06, $P=0.09)$. This implied that both $P$. vivax and $P$. falciparum infections could present with bleeding episodes should there be a change in platelet counts in the infected patients.

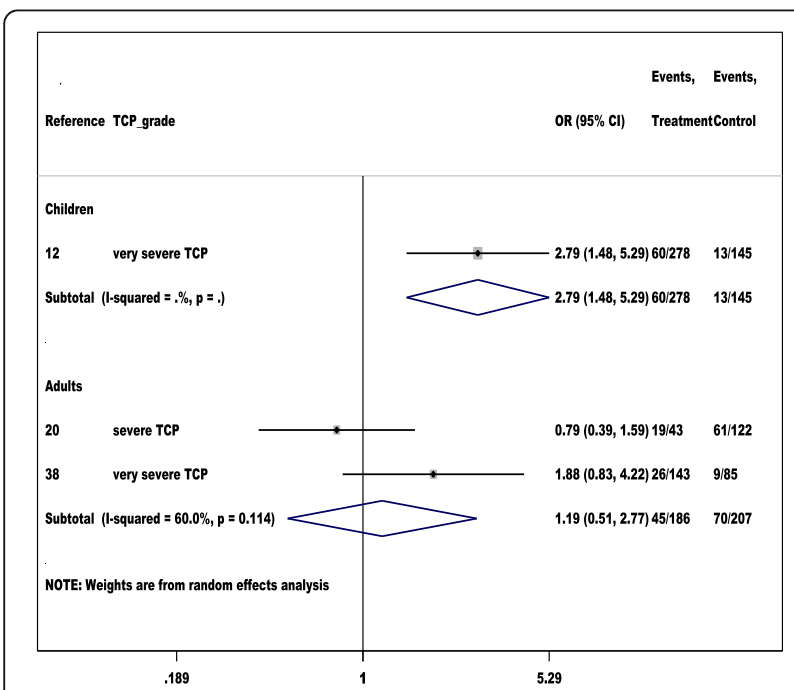

Fig. 3 Comparison of thrombocytopaenia in patients with vivax malaria and with falciparum malaria. OR ( $95 \%$ C): odds ratio and corresponding $95 \%$ confidence interval. The middle vertical line is OR 1, indicating a null value. The horizontal line indicates study specific odds ratio and corresponding $95 \%$ confidence interval. $P$ value is not indicated by default, when $R^{2}$ value becomes $0 \%$. Very severe TCP denotes very severe thrombocytopenia; severe TCP denotes severe thrombocytopenia
Associated risks of thrombocytopaenia in $P$. vivax malaria Three studies with PCR confirmed $P$. vivax malaria reported parasite density. Of these, one study showed a higher parasite density among patients with severe thrombocytopaenia in $P$. vivax malaria than in those with either mild thrombocytopaenia or no thrombocytopaenia $(P=0.524)$ [26]. The remaining 2 studies [21, 38] qualitatively reported almost the same $P$. vivax parasite density in patients with thrombocytopaenia and those without thrombocytopaenia. This was indirectly supported by another study with 456 non-PCR confirmed P. vivax, showing no statistically significant relationship between parasite density and thrombocytopaenia $\left(R^{2}=0\right)$. This implied that parasite load was not a predictor of change in platelet counts in these patients [39].

One study showed a significantly lower haemoglobin (Hb) level with severe thrombocytopaenia (11.66 \pm 2.19 , $n=79$ ) compared to those with moderate thrombocytopaenia $(12.65 \pm 1.63, n=93) \quad(P=0.002)$ or the healthy (non-malaria) participants $(12.29 \pm 1.99, n=100)$ $(P=0.05)$ [58]. This indicated that as the platelet count decreased, so did the $\mathrm{Hb}$ level in patients with $P$. vivax malaria. However, a small study reported that there was no significant difference in $\mathrm{Hb}$ levels between $P$. vivax malaria with severe thrombocytopaenia $(n=10)$ and those with mild thrombocytopaenia $(n=45) \quad(13.00 \pm 3.25$ vs $12.90 \pm 3.25)(P=0.9)$ or no thrombocytopaenia (normal platelet counts $)(n=35) \quad(13.00 \pm 3.25$ vs $12.10 \pm 3.25)$ $(P=0.4)$ [26]. The same study on patients with $P$. vivax malaria reported that there were higher urea levels in patients with severe thrombocytopaenia $(n=10)$ compared to patients with mild thrombocytopaenia $(n=45)(41.50 \pm$ 10.37 vs $30.00 \pm 7.20)(P<0.001)$ or no thrombocytopaenia $(n=35) \quad(41.50 \pm 10.37$ vs $28.00 \pm 7.00) \quad(P<0.001) \quad[26]$. Also, there was higher serum bilirubin levels in patients 
with severe thrombocytopaenia $(n=10)(1.70 \pm 0.42)$ compared to those with mild thrombocytopaenia $(n=45)$ $(1.30 \pm 0.32)(P=0.001)$ or no thrombocytopaenia $(n=35)$ $(0.90 \pm 0.25) \quad(P<0.001) \quad[26]$. Therefore, a decreasing platelet count was related to both elevated urea and serum bilirubin levels in P. vivax infection. A study on 51 children in India had reported that the association of severe $P$. vivax malaria in children with severe thrombocytopaenia was 2.6 times significantly greater than those with non-severe thrombocytopaenia (OR: 2.60, 95\% CI: 1.20-5.52, $P \leq 0.014$ ) [12].

One study has reported that anaemia with thrombocytopaenia was more common in patients with $P$. falciparum malaria $(17.6 \%, 13 / 74$ vs $39 / 103)$ compared to those with $P$. vivax malaria $(37.9 \%, 39 / 103)$ $(P=0.004)[40]$.

\section{Mortality risk}

A pooled analysis of 2 studies with PCR confirmed parasite species [40,64] showed an equal risk of mortality with severe thrombocytopaenia in $P$. vivax malaria patients $(10.2 \%, 5 / 49)$ and $P$. falciparum malaria patients $(14 \%, 8 / 57) \quad(O R: 1.16, \quad 95 \% \quad C I: 0.30-4.60, \quad P=0.87)$ (Additional file 8). Another study reported a 9-fold increase in the risk of death from $P$. vivax malaria in children with severe anaemia and severe thrombocytopaenia, compared to those without anaemia or thrombocytopaenia (OR: 9.21, 95\% CI: 4.53-18.73) [11].

\section{Discussion}

Based on available data from 58 individual observational studies across 12 endemic countries, the current study has provided some evidence of the increasing risks of developing thrombocytopaenia in $P$. vivax malaria patients. The observational design in these studies was appropriate as it quantified the frequency and associated risks in regular clinical practice, reflecting real life situations. The findings suggest that thrombocytopaenia is a common manifestation equally observed in both human $P$. vivax and $P$. falciparum infections. A review of studies restricted to Brazil reported that the mortality rate in $P$. vivax malaria with severe thrombocytopaenia alone was comparable to that of $P$. falciparum malaria [4]. Our findings also suggest that both the magnitude of severe thrombocytopaenia and mortality risks are similar in both $P$. falciparum and P. vivax malaria. Dating back to the era of induced malaria therapy, the fatality rate with the Madagascar strain of P. vivax was $10-15 \%$ when used to treat patients with neurosyphilis in the United Kingdom [90]. Details of this evidence are available elsewhere [80].

Although the mechanism of thrombocytopaenia in malaria is not fully understood, numerous studies have hypothesied possible patho-immunological mechanisms of thrombocytopaenia in malaria. Oxidative stress [23], macrophage colony stimulating factor [91], immunoglobulin $\mathrm{G}$ binding to platelet-bound malaria antigens [73], spleen pooling [92], increased plasma cell free circulating nucleic acids levels in $P$. vivax [93] and platelet phagocytosis [21] are possible mechanisms resulting in damage to thrombocytes or the excessive removal of platelets. A case study in India reported haemophagocytosis occurring in a $P$. vivax malaria patient with a platelet count $16000 / \mathrm{mm}^{3}$ [87]. Moreover, an interaction between Plasmodium and platelets has been postulated as $P$. vivax has been seen within the platelets of patients when using electron microscopy [94]. It is probable that thrombocytopaenia in malaria is actually a multifactorial phenomenon [21].

In our review, we found that $10.1 \%$ of $P$. vivax infected patients had severe to very severe thrombocytopaenia, regardless of confirmatory tests used for $P$. vivax malaria. Of note, a large-scale study in Indonesia [11] had contributed to this pooled proportion, and there the prevalence was $8.4 \%$ (1650/19 552). An earlier review reported that the pooled proportion of severe thrombocytopaenia was $7.5 \%$ (95\% CI: 4.2-10.8\%) amongst outpatients with $P$. vivax malaria [95]. This difference might be due to the periods covered by the included studies, variation in selection criteria used by the reviews and/or levels of malaria endemicity in the study areas. Moreover, the proportion of thrombocytopaenia will be higher in studies performed at tertiary-referral hospitals (with a higher proportion of patients with severe vivax malaria), and lower in community-based studies. In our analysis, we included published studies between 1988 and 2015. We did not include studies with imported malaria cases $[72,79,89]$ nor those with pregnant women included [76, 78]. The difference in the proportion of severe thrombocytopaenia between the current review and earlier review might also be due to the drug resistance status of parasites in the study areas in the included studies. It has been found that drug resistant parasites prolong their stay in their hosts and do more damage, resulting in thrombocytopaenia [96].

\section{Clinical relevance}

A limited number of studies included in this review documented that low $\mathrm{Hb}$, and high serum bilirubin and urea levels in $P$. vivax malaria were associated with severe thrombocytopaenia. These findings implied that severe anaemia, and impaired liver and renal functions would be expected in patients with severe thrombocytopaenia and $P$. vivax malaria. This also indicates that MOD may occur in malaria patients with thrombocytopaenia. This is a similar phenomenon to that seen in $P$. falciparum infections. This provides further evidence to refute the long-held view that $P$. vivax infection is benign $[5,11$, 
$38,39,80]$. This misconception and may have led to inappropriate clinical management, research and control [63] of P. vivax malaria. This collective evidence supports the need for increased efforts to actively control and then eliminate both $P$. vivax [38] and $P$. falciparum infections.

This review reported minor bleeding episodes. Thrombocytopaenia, even in severe cases, was not commonly accompanied with severe bleeding [11, 24] or clinical bleeding in malaria [12]. This might be due to the good tolerance of a low platelet count in malaria patients through an enhanced aggregability pathway [49]. Another possibility is that larger platelets observed in thrombocytopaenic patients may compensate for the low number of platelets in the periphery, thereby preserving primary haemostasis and avoiding severe bleeding [24]. The high proportion of severe thrombocytopaenia in $P$. vivax malaria patients found in this review highlights the clinical relevance of this parameter in diagnosis and treatment. However, in the absence of (severe) bleeding manifestations in malaria patients, thrombocytopaenia alone is not regarded as a WHO criterion used to define severe malaria [8]. Additionally, other severe manifestations in the form of impaired renal and liver function, leading to MOD and increased mortality have been documented in studies from India [39] and Brazil [11] and further highlight the need for a broadened clinical awareness of $P$. vivax malaria and its complications. Although it was not included in the current analysis, a case study in India had reported retinal haemorrhage in a patient of $P$. vivax malaria who had a platelet count of $40000 / \mathrm{mm}^{3}$ [86].

\section{Study limitations}

The present study has several limitations. Not all studies included in this review used the same methods for platelet 'labelling'. Hence, misclassification bias could potentially affect the interpretation of the results. For instance, the haemolysis necessary to count platelets in the chamber creates pseudo-platelets [97], resulting in erroneously high numbers of platelets being counted. This shortfall is avoided if an electronic counter is used in which platelets are counted in platelet rich, red blood cell free, and parasite-free plasma [72]. Moreover, semiimmune malaria patients were likely to have low parasite counts and may not have an increase in platelet count even after treatment [72].

We did not undertake segregated analysis of thrombocytopaenia by use or not of PCR confirmed speciation. This is because microscopy by technicians at the malaria clinics in the endemic countries may contribute to the relatively minor possible errors. Moreover, PCR in the studies included in the current review might have excluded errors made by microscopists in the same studies. Although the quality of the studies included was variable if $P$. vivax malaria was not confirmed by PCR, the current review attempted to minimize this problem by including studies which focused on specific groups in endemic areas where $P$. vivax is prevalent. This reinforces our confidence in the effect estimates. Nevertheless, the current work focused exclusively on thrombocytopaenia and also reported comparisons between $P$. vivax and $P$. falciparum malaria other than solely giving frequencies of each disease thereby yielding more reliable and comprehensive information.

We were unable to rule out association of co-infections such as intestinal parasitic infections [58], acute parvovirus B19 infection [98] or an endemic viral infection such as dengue in Brazil [4]. These comorbid infections in patients included in the primary studies could contribute to a substantial percentage of thrombocytopaenia [4]. One published study highlighted that acute or chronic comorbidities were detected in $58 \%(14 / 24)$ of the patients with P. vivax in the Western Brazilian Amazon [99]. Another confounding factor may occur if patients in the primary studies had taken primaquine-containing antimalarial treatment, as they might have recovered normal platelets counts. Hence, the interpretation of the current findings needs to consider potential effects of co-infections and antimalarial medication as confounding factors.

The manifestations of thrombocytopaenia could also be influenced by context-dependent factors such as the level of malaria endemicity, accessibility to effective malaria treatment and the age-specific and gender-specific host immunity. We have found that a variation in the risk of developing thrombocytopaenia in $P$. vivax malaria between children and adults might be due to a difference in immune status between the age groups. For instance, young children exhibit an "antidisease immunity" that affects the risk and extent of morbidity associated with a given parasite density [100]. Inadequate data in the included studies preclude the ability to perform stratified analyses based on any of these potential influencing factors.

\section{Implications}

Although further substantiation is required, one study reported that patients with mild thrombocytopaenia were about 32 times more likely to have malaria infection than those with normal platelet count (OR: 31.8, 95\% CI: 25.1-39.7, $P \leq 0.001)$ [41]. An estimation of platelet counts is readily available with an automated blood counter even in some remote health posts, where other biochemical and inflammatory markers are not available. The presence of thrombocytopaenia in people from malaria endemic areas may also be useful to support the diagnosis of malaria in cases with low levels of parasite biomass [41], as it is common in natural $P$. 
vivax infections. Since severe thrombocytopaenia may be associated with several infectious diseases such as dengue and leptospirosis, patients with persistent thrombocytopaenia in malaria endemic areas should also be thoroughly investigated for co-infections or other identifiable causes of thrombocytopaenia.

The association of severe malaria was found to be stronger with severe thrombocytopaenia in children with a specificity of $88.3 \%$ and a positive predictive value of $85 \%$ [12]. Therefore, the presence of severe thrombocytopaenia should serve as a warning sign of poor outcome, particularly when coexisting with severe anaemia [11]. Any patient with an acute febrile illness without localized signs and having a combination of anaemia and thrombocytopaenia should raise the possibility of malaria infection, and they should then proceed to use confirmatory tests [12]. The increased mortality risk in vivax malaria cases with severe thrombocytopaenia as well as severe anaemia highlights the need for healthcare personnel to promptly examine for these signs in malaria patients living in endemic areas.

Based on the findings, patients with severe thrombocytopaenia in malaria endemic settings and without other definitive causes for the thrombocytopaenia should be treated as severe malaria [12] and the presence of severe thrombocytopaenia should be included as a defining criterion of malaria severity for both $P$. falciparum and $P$. vivax malaria [11]. The platelet count (indicating the presence of thrombocytopaenia) coupled with other clinical and microscopy parameters can significantly improve malaria diagnosis, provide timely treatment for malaria infections [41] and address the associated higher morbidity and mortality risks.

\section{Conclusion}

The current review has provided some evidence of the clinical relevance of severe thrombocytopaenia in $P$. vivax malaria. To substantiate these findings, there is a need for future well designed, large-scale, prospective studies among patients infected with $P$. vivax from different countries and epidemiological settings with various age and gender groups represented.

\section{Additional files}

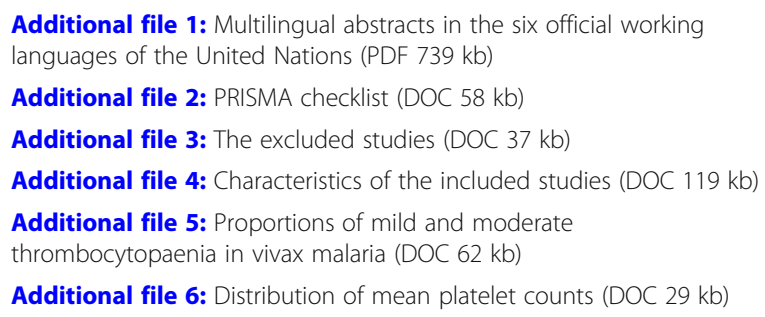

Additional file 7: Proportion of bleeding manifestation in patients with vivax malaria (DOC $32 \mathrm{~kb}$ )

Additional file 8: Comparison of mortality with severe thrombocytopaenia between severe vivax malaria and severe falciparum malaria (PDF $99 \mathrm{~kb}$ )

\section{Abbreviations}

AJOL: African Journals Online; Hb: Hemoglobin; LILACS: Latin American and Caribbean Health Sciences Literature; MD: Mean difference; MOD: Multi-organ dysfunction; OR: Odds ratio; P. falciparum: Plasmodium falciparum; $P$. vivax: Plasmodium vivax; RDT: Rapid onsite diagnostic test; RR: Risk ratio; SD: Standard deviation; TBF: Thick/thin blood film

\section{Acknowledgements}

The authors thank the patients and researchers of the primary studies. We thank our institutions for allowing us to perform this study. Our thankful appreciation is extended to the two anonymous reviewers and editors for the comments provided and the valuable inputs to improve the quality of this manuscript. CN is grateful to Professor Joon Wah Mak for technical inputs to an earlier version and Professor Frederick Smales for supporting English edit in the revised version.

\section{Funding}

None.

\section{Availability of data and materials}

The data supporting the findings can be found in the main paper and an additional supporting file.

\section{Authors' contributions}

CN: designed the study, collected data, carried out the statistical analysis, interpreted the results and wrote the manuscript. MAW: conceptualized, participated in its design, interpreted the results and assisted in writing the manuscript. Both authors read and approved the final manuscript.

\section{Ethics approval and consent to participate}

Not applicable.

\section{Consent for publication}

Not applicable.

\section{Competing interests}

The authors declare that they have no competing interests.

Received: 3 August 2017 Accepted: 24 January 2018

Published online: 09 February 2018

\section{References}

1. Price RN, Tjitra E, Guerra CA, Yeung S, White NJ, Anstey NM. Vivax malaria: neglected and not benign. Am J Trop Med Hyg. 2007;77:79-87.

2. WHO. Global technical strategy for malaria 2016-2030. Geneva: World Health Orgnization; 2015.

3. Howes RE, Battle KE, Mendis KN, Smith DL, Cibulskis RE, Baird JK, et al. Global epidemiology of plasmodium vivax. Am J Trop Med Hyg. 2016;95: 15-34.

4. Lacerda MV, Mourao MP, Alexandre MA, Siqueira AM, Magalhaes BM, Martinez-Espinosa FE, et al. Understanding the clinical spectrum of complicated Plasmodium vivax malaria: a systematic review on the contributions of the Brazilian literature. Malar J. 2012;11:12.

5. McMorran BJ, Marshall VM, De Graaf C, Drysdale KE, Shabbar M, Smyth GK, et al. Platelets kill intraerythrocytic malarial parasites and mediate survival to infection. Science. 2009;323:797-800.

6. McMorran BJ, Burgio G, Foote SJ. New insights into the protective power of platelets in malaria infection. Commun Integer Biol. 2013;6:e23653.

7. Thapa R, Biswas B, Mallick D, Sardar S, Modak S. Childhood plasmodium vivax malaria with severe thrombocytopenia and bleeding manifestations. J Pediatr Hematol Oncol. 2009;31:758-9.

8. WHO. Definitions: severe falciparum malaria. In: guidelines for the treatment of malaria. 3rd ed. Geneva: World Health Orgnization; 2015. 
9. Hanson J, Phu NH, Hasan MU, Charunwatthana P, Plewes K, Maude RJ, et al. The clinical implications of thrombocytopenia in adults with severe falciparum malaria: a retrospective analysis. BMC Med. 2015;13:97.

10. Moher D, Liberati A, Tetzlaff J, Altman DG, Grp P. Preferred reporting items for systematic reviews and meta-analyses: the PRISMA statement. PLoS Med. 2009:6:e1000097.

11. Lampah DA, Yeo TW, Malloy M, Kenangalem E, Douglas NM, Ronaldo D, et al. Severe thrombocytopenia: a risk factor for mortality in Papua, Indonesia. J Infect Dis. 2015;211:623-34.

12. Tanwar GS, Khatri PC, Chahar CK, Sengar GS, Kochar A, Tanwar G, et al. Thrombocytopenia in childhood malaria with special reference to P. Vivax Monoinfection: a study from Bikaner (northwestern India). Platelets. 2012;23:211-6.

13. Greenland S, Thomas DC, Morgenstern $\mathrm{H}$. The rare-disease assumption revisited. A critique of estimators of relative risk for case-control studies. Am J Epidemiol. 1986:124:869-983.

14. Hozo S, Djulbegovic B, Hozo I. Estimating the mean and variance from the median, range, and the size of a sample. BMC Med Res Methodol. 2005:5:13.

15. Higgins JPT, Green S. Cochrane handbook for systematic reviews of interventions version 5.1.0. The Cochrane collaboration, 2011.

16. Aatif $\mathrm{S}$, Jamal $\mathrm{Q}$, Altaf $\mathrm{A}$, Salimullah. Is vivax malaria really benign? - a Karachi-based study. J Pak Med Assoc. 2013;63:721-4.

17. Araujo CF, Lacerda MV, Abdalla DS, Lima ES. The role of platelet and plasma markers of antioxidant status and oxidative stress in thrombocytopenia among patients with vivax malaria. Mem Inst Oswaldo Cruz. 2008;103(6): 517-21.

18. Arevalo-Herrera M, Lopez-Perez M, Medina L, Moreno A, Gutierrez JB, Herrera S. Clinical profile of Plasmodium falciparum and Plasmodium vivax infections in low and unstable malaria transmission settings of Colombia. Malar J. 2015;14:154

19. Asma U, Taufiq F, Khan W. Prevalence and clinical manifestations of malaria in Aligarh, India. Korean J Parasitol. 2014;52:621-9.

20. Barber BE, William T, Grigg MJ, Menon J, Auburn S, Marfurt J, et al. A prospective comparative study of knowlesi, falciparum, and vivax malaria in Sabah, Malaysia: high proportion with severe disease from plasmodium knowlesi and plasmodium vivax but no mortality with early referral and artesunate therapy. Clin Infect Dis. 2013:56:383-97.

21. Coelho HCC, Lopes SCP, Pimentel JPD, Nogueira PA, Costa FTM, et al. Thrombocytopenia in plasmodium vivax malaria is related to platelets phagocytosis. PLoS One. 2013;8:e63410.

22. Dhungat MP, Dhungat PP. Thrombocytopenia in patients of malaria correlation with type ofMalaria and it's clinical significance. Online International Interdisciplinary Research Journal. 2013;3:21-6.

23. Erel O, Vural H, Aksoy N, Aslan G, Ulukanligil M. Oxidative stress of platelets and thrombocytopenia in patients with vivax malaria. Clin Biochem. 2001; 34:341-4.

24. Erhart LM, Yingyuen K, Chuanak N, Buathong N, Laoboonchai A, Miller RS, et al. Hematologic and clinical indices of malaria in a semi-immune population of western Thailand. Am J Trop Med Hyg. 2004;70:8-14.

25. George P, Alexander LM. A study on the clinical profile of complicated plasmodium vivax mono-infections. Asian Pac J Trop Med. 2010;3:560-2.

26. Gomes LT, Alves-Junior ER, Rodrigues-Jesus C, Nery AF, Gasquez-Martin TO, et al. Angiopoietin-2 and angiopoietin-2/angiopoietin-1 ratio as indicators of potential severity of plasmodium vivax malaria in patients with thrombocytopenia. PLoS One. 2014;9:e109246.

27. Gonçalves RM, Salmazi KC, Santos BA, Bastos MS, Rocha SC, Boscardin SB, et al. CD4+ CD25+ Foxp3+ regulatory T cells, dendritic cells, and circulating cytokines in uncomplicated malaria: do different parasite species elicit similar host responses? Infect Immun. 2010;8:4763-72.

28. Goyal JP, Makwana AM. Comparison of clinical profile between P. Vivax and P. Falciparum malaria in children: a tertiary care centre perspective from India. Malar Res Treat. 2014;2014:132672

29. Gupta NK, Bansal SB, Jain UC, Sahare K. Study of thrombocytopenia in patients of malaria. Trop Parasitol. 2013;3:58-61.

30. Haroon H, Fazel PA, Naeem M, Mobin A, Naqvi AH, Makki K. Hide and seek: hematological aspects of malaria-a developing country perspective. J Infect Dev Ctries. 2013;7:273-9.

31. Jadhav UM, Patkar VS, Kadam NN. Thrombocytopenia in malaria-correlation with type and severity of malaria. J Assoc Physicians India. 2004;52:615-8.

32. Kaushik JS, Gomber S, Dewan P. Clinical and epidemiological profiles of severe malaria in children from Delhi,India. J Health Popul Nutr. 2012; 30:113-6.
33. Khan SJ, Abbass Y, Marwat MA. Thrombocytopenia as an indicator of malaria in adult population. Malar Res Treat. 2012;405981:4.

34. Khan W, Zakai HA, Umm-E-Asma. Clinico-pathological studies of plasmodium falciparum and Pasmodium vivax-malaria in India and Saudi Arabia. Acta Parasitol. 2014;59:206-12.

35. Kim JS, Oh JS, Chang EA, Bae SY, Nam DH, Lee CH, et al. Alteration of platelet counts and lipid profiles after treatment of acute Plasmodium vivax. Acta Trop. 2008;106:39-43.

36. Kochar DK, Das A, Kochar SK, Saxena V, Sirohi P, Garg S, et al. Severe Plasmodium vivax malaria: a report on serial cases from Bikaner in northwestern India. Am J Trop Med Hyg. 2009;80:194-8.

37. Kochar DK, Das A, Kochar A, Middha S, Acharya J, Tanwar GS, et al. Thrombocytopenia in Plasmodium falciparum, Plasmodium vivax and mixed infection malaria: a study from Bikaner (northwestern India). Platelets. 2010:21:623-7.

38. Kochar DK, Tanwar GS, Khatri PC, Kochar SK, Sengar GS, Gupta A, et al. Clinical features of children hospitalized with malaria-a study from Bikaner, Northwest India. Am J Trop Med Hyg. 2010;83:981-9.

39. Kochar DK, Tanwar GS, Agrawal R, Kochar S, Tanwar G, Falodia SK, et al. Platelet count and parasite density: independent variable in Plasmodium vivax malaria. J Vector Borne Dis. 2012;49:191-2.

40. Kochar DK, Das A, Kochar A, Middha S, Acharya J, Tanwar GS. A prospective study on adult patients of severe malaria caused by Plasmodium falciparum, Plasmodium vivax and mixed infection from Bikaner, Northwest India. J Vector Borne Dis. 2014;51:200-10.

41. Kotepui M, Phunphuech B, Phiwklam N, Chupeerach C, Duangmano S. Effect of malarial infection on haematological parameters in population near Thailand-Myanmar border. Malar J. 2014;13:218.

42. Kwak YG, Lee HK, Kim M, Um TH, Cho CR. Clinical characteristics of vivax malaria and analysis of recurred patients. Infect Chemother. 2013;45:69-75.

43. Leal-Santos FA, Silva SB, Crepaldi NP, Nery AF, Martin TO, Alves-Junior ER, et al. Altered platelet indices as potential markers of severe and complicated malaria caused by Plasmodium vivax: a cross-sectional descriptive study. Malar J. 2013;12:462.

44. Limaye CS, Vikram A, Londhey VA, Nabar ST. The study of complications of vivax malaria in comparison with falciparum malaria in Mumbai. J Assoc Physicians India. 2012;60:15-8.

45. Mahgoub H, Gasim Gl, Musa IR, Adam I. Severe plasmodium vivax malaria among Sudanese children at new Halfa hospital, eastern Sudan. Parasit Vectors. 2012:5:154.

46. Manning L, Laman M, Law I, Bona C, Aipit S, Teine D, et al. Features and prognosis of severe malaria caused by Plasmodium falciparum, Plasmodium vivax and mixed Plasmodium species in Papua new Guinean children. PLoS One. 2011:6:e29203.

47. Martinez-Salazar EL, Tobon-Castano A. Platelet profile is associated with clinical complications in patients with vivax and falciparum malaria in Colombia. Rev Soc Bras Med Trop. 2014;47:341-9.

48. Mehmood A, Ejaz K, Ahmed T. Severity of Plasmodium vivax malaria in Karachi: a cross-sectional study. J Infect Dev Ctries. 2012;6:664-70.

49. Mohanty D, Marwaha N, Ghosh K, Sharma S, Garewal G, Shah S, et al. Functional and ultrastructural changes of platelets in malarial infection. Trans R Soc Trop Med Hyg. 1988:82:369-75.

50. Mohanty D, Ghosh K, Nandwani SK, Shety S, Philips C, Rizvi S, et al. Fibrinolysis, inhibitors of blood coagulation, and monocyte derived coagulant activity in acute malaria. Am J Hematol. 1997;54:23-9.

51. Mohapatra MK, Dashet LK, Mohapatra A. Severe vivax malaria: a study on its cinical manifestations, risk factors, outcome and therapeutic efficacy of artesunate. Int J Clin Case Report. 2013;3:17-25.

52. Nadkar MY, Huchche AM, Singh R, Pazare AR. Clinical profile of severe plasmodium vivax malaria in a tertiary care centre in Mumbai from June 2010-January 2011. J Assoc Physicians India. 2012; 60:11-3.

53. Naha K, Dasari S, Prabhu M. Spectrum of complications associated with Plasmodium vivax infection in a tertiary hospital in south-western India. Asian Pac J Trop Med. 2012:75-82.

54. Oh MD, Shin H, Shin D, Kim U, Lee S, Kim N, et al. Clinical features of vivax malaria. Am J Trop Med Hyg. 2001;65:143-6.

55. Poespoprodjo JR, Fobia W, Kenangalem E, Lampah DA, Hasanuddin A, Warikar $\mathrm{N}$, et al. Vivax malaria: a major cause of morbidity in early infancy. Clin Infect Dis. 2009;48:1704-12. 
56. Rajeshwar K, Karibasappa BG. Clinical profile of severe Plasmodium vivax malaria in a tertiary centre in J.J.M. Medical college, Davangere. Indian J Basic Appl Med Res. 2015;4:133-9.

57. Rasheed A, Saeed S, Khan SA. Clinical and laboratory findings in acute malaria caused by various plasmodium species. J Pak Med Assoc. 2009; 59:220-3.

58. Raza A, Khan MS, Ghanchi NK, Raheem A, Beg MA. Tumour necrosis factor, interleukin-6 and interleukin-10 are possibly involved in Plasmodium vivaxassociated thrombocytopaenia in southern Pakistani population. Malar J. 2014;13:323.

59. Rizvi I, Tripathi DK, Chughtai AM, Beg M, Zaman S, Zaidi N. Complications associated with plasmodium vivax malaria: a retrospective study from a tertiary care hospital based in western Uttar Pradesh, India. Ann Afr Med. 2013;12:155-9.

60. Rodrigues-da-Silva RN, Lima-Junior JDC, Fonseca BDP, Antas PRZ, Baldez A Storer FL, et al. Alterations in cytokines and haematological parameters during the acute and convalescent phases of Plasmodium falciparum and Plasmodium vivax infections. Mem Inst Oswaldo Cruz. 2014;109:154-62.

61. Sarkar D, Ray S, Saha M, Chakraborty A, Talukdar A. Clinico-laboratory profile of severe plasmodium vivax malaria in a tertiary care centre in Kolkata. Trop Parasitol. 2013:3:53-7.

62. Saravu K, Docherla M, Vasudev A, Shastry BA. Thrombocytopenia in vivax and falciparum malaria: an observational study of 131 patients in Karnataka, India. Ann Trop Med Parasitol. 2011;105:593-8.

63. Singh H, Parakh A, Basu S, Rath B. Plasmodium vivax malaria: is it actually benign? J Infect Public Health. 2011;4:91-5.

64. Shaikh S, Memon H, Lohano B, Shaikh A, Ahmed I, lohano B, et al. Severe disease in children hospitalized with a diagnosis of Plasmodium vivax in south-eastern Pakistan. Malar J. 2012;11:144.

65. Singh J, Purohit B, Desai A, Savardekar L, Shanbag P, Kshirsagar N. Clinical manifestations, treatment, and outcome of hospitalized patients with Plasmodium vivax malaria in two Indian states: a retrospective study. Malar Res Treat. 2013:341862.

66. Singh A, Goyal M, Sharma D. Thrombocytopenia in malaria: its usefulness in a clinical setting. A study from western Rajasthan, India. Trop Dr. 2014;44: 205-8.

67. Song HH, SO O, Kim SH, Moon SH, Kim JB, Yoon JW, et al. Clinical features of plasmodium vivax malaria. Korean J Intern Med. 2003;18:220-4.

68. Srivastava S, Ahmad S, Shirazi N, Kumar Verma S, Puri P. Retrospective analysis of vivax malaria patients presenting to tertiary referral centre of Uttarakhand. Acta Trop. 2011;117:82-5.

69. Taylor WR, Widjaja H, Basri H, Ohrt C, Taufik T, Tjitra E, et al. Changes in the total leukocyte and platelet counts in Papuan and non-Papuan adults from northeast Papua infected with acute Plasmodium vivax or uncomplicated Plasmodium falciparum malaria. Malar J. 2008;18(7):259.

70. Uttra MK, Devrajani BR, Shaikh K, Shaikh KR, Shah SZA. Severity of thrombocytopenia and prolonged bleeding time in patients with malaria (a clinical study of 162 malaria cases). World Appl Sci J. 2010;9:484-8.

71. Vaidya KKA, Vernekar P. Thrombocytopenia a in relation with plasmodium vivax malaria. J Evol Med Dental Sci. 2012;1:413.

72. Horstmann RD, Dietrich M, Bienzle U, Rasche H. Malaria-induced thrombocytopenia. Blutalkohol. 1981;42:157-64.

73. Kelton JG, Keystone J, Denomme G, Tozman E, Glynn M, Neame PB, et al. Immune-mediated thrombocytopenia of malaria. J Clin Invest. 1983;71:832-6.

74. Gerardin P, Rogier C, Ka AS, Jouvencel P, Brousse V, Imbert P. Prognostic value of thrombocytopenia in African children with falciparum malaria. Am J Trop Med Hyg. 2002;66:686-91.

75. Park JW, Park SH, Yeom JS, Huh AJ, Cho YK, Ahn JY, et al. Serum cytokine profiles in patients with Plasmodium vivax malaria: a comparison between those who presented with and without thrombocytopenia. Ann Trop Med Parasitol. 2003;97:339-44

76. Rodriguez-Morales AJ, Sanchez E, Vargas M, Piccolo C, Colina R, Arria M, et al. Occurrence of thrombocytopenia in Plasmodium vivax malaria. Clin Infect Dis. 2005:41:130-1.

77. Kochar D, Saxena V, Singh N, Kochar S, Kumar V, Das A. Plasmodium vivax malaria. Emerg Infect Dis. 2005;11:132-4.

78. Tan SO, McGready R, Zwang J, Pimanpanarak M, Sriprawat K, Thwai KL, et al. Thrombocytopenia in pregnant women with malaria on the Thai-Burmese border. Malar J. 2008;7:209.
79. Khan FY, Lutof AK, Yassin MA, Khattab MA, Saleh M, Rezeq HY, Almaslamani M. Imported malaria in Qatar: a one year hospital-based study in 2005. Travel Med Infect Dis. 2009;7:111-7.

80. Baird JK. Evidence and implications of mortality associated with acute Plasmodium vivax malaria. Clin Microbiol Rev. 2013;26:36-57.

81. Faseela TS, Roche RA, Anita KB, Malli CS, Rai Y. Diagnostic value of platelet count in malaria. J Clin Diagn Res. 2011;5:464-6.

82. Lampah DA, Yeo TW, Hardianto SO, Tjitra E, Kenangalem E, Sugiarto P, et al. Coma associated with microscopy-diagnosed Plasmodium vivax: a prospective study in Papua, Indonesia. PLoS Negl Trop Dis. 2011;5:e1032

83. Garg S, Saxena V, Lumb V, Pakalapati D, Boopathi PA, Subudhi AK, et al. Novel mutations in the antifolate drug resistance marker genes among Plasmodium vivax isolates exhibiting severe manifestations. Exp Parasitol. 2012;132:410-6

84. Campos FMF, Santos MLS, Kano FS, Fontes CJF, Lacerda MVG, Brito CFA et al. Genetic variability in platelet integrin a $2 \beta 1$ density: possible contributor to plasmodium vivax-induced severe thrombocytopenia. Am J Trop Med Hyg. 2013;88:325-8.

85. Kochar A, Kalra P, Kochar S, Kochar SK, Kochar DK. Retinal haemorrhage: an unusual presentation of vivax malaria. J Vector Borne Dis. 2013:50:321-2.

86. Raza A, Ghanchi NK, Zbairi AS, Raheem A, Nizami S, Beg MA. Tumor necrosis factor-a, interleukin-10, intercellular and vascular adhesion molecules are possible biomarkers of disease severity in complicated Plasmodium vivax isolated from Pakistan. PLoS One. 2013:8:e81363.

87. Tanwar GS, Lahoti A, Tanwar P, Agrawal R, Khatri PC, Kochar DK. Hemophagocytosis syndrome associated with severe Plasmodium vivax malaria in a child in Bikaner (northwestern India). J Vector Borne Dis. 2013; 50:318-20.

88. O'Brien AT, Ramírez JF, Martínez SP. A descriptive study of 16 severe Plasmodium vivax cases from three municipalities of Colombia between 2009 and 2013. Malar J. 2014:13:404.

89. Antinori S, Corona A, Ridolfo AL, Galimberti L, Ricaboni D, Milazzo L, et al. Imported Plasmodium vivax malaria with severe thrombocytopenia: can it be severe malaria or not? Malar J. 2016;15:105

90. Nicol WD. The relation of syphilis to mental disorder and the treatment of G.P.I. By malaria. Br J Vener Dis. 1933;9:219-29.

91. Lee SH, Looareesuwan S, Chan J, Wilairatana P, Vanijanonta S, Chong SM, Chong BH. Plasma macrophage colony-stimulating factor and P-selectin levels in malaria-associated thrombocytopenia. Thromb Haemost. 1997;77: 289-93.

92. Skudowitz RB, Katz J, Lurie A, Levin J, Metz J. Mechanisms of thrombocytopenia in malignant tertian malaria. Br Med J. 1973;2:515-8.

93. Franklin BS, Vitorino BLF, Coelho HC, Menezes-Neto A, Santos MLS, Campos FMF, et al. Plasma circulating nucleic acids levels increase according to the morbidity of Plasmodium vivax malaria. PLoS One. 2011;6:e19842.

94. Fajardo LF, Tallent C. Malarial parasites within human platelets. JAMA. 1974; 229:1205.

95. Rahimi BA, Thakkinstian A, White NJ, Sirivichayakul C, Dondorp AM Chokejindachai W. Severe vivax malaria: a systematic review and metaanalysis of clinical studies since 1900. Malar J. 2014;13:481.

96. Tjitra E, Anstey NM, Sugiarto P, Warikar N, Kenangalem E, Karyana M, et al. Multidrug-resistant Plasmodium vivax associated with severe and fatal malaria: a prospective study in Papua, Indonesia. PLoS Med. 2008;5:e128.

97. Maina RN, Walsh D, Gaddy C, Hongo G, Waitumbi J, Otieno L, et al. Impact of Plasmodium falciparum infection on haematological parameters in children living in western Kenya. Malar J. 2010;9:S4

98. Wildig J, Michon P, Siba P, Mellombo M, Ura A, Muelle I, et al. Parvovirus B19 infection contributes to severe anemia in young children in Papua New Guinea. J Infect Dis. 2006:194:146-53.

99. Lanca EF, Magalhaes BML, Vitor-Silva S, Siqueira AM, Benzecry SG, et al. Risk factors and characterization of Plasmodium vivax-associated admissions of pediatric intensive care units in the Brazilian Amazon. PLoS One. 2012;7: e35406.

100. Doolan DL, Dobano C, Baird JK. Acquired immunity to malaria. Clin Microbiol Rev. 2009:22:13-36. 